\title{
HAPLOID SELECTION IN “DIPLOID” ORGANISMS
}

Simone Immler ${ }^{1}$

5

${ }^{1}$ School of Biological Sciences, University of East Anglia, Norwich Research Park, Norwich, NR4 7TJ, UK

Email: s.immler@uea.ac.uk

Short title: Haploid selection in diploid organisms

Keywords: ploidy, gametic selection, biphasic life cycle, sex chromosomes, 15 imprinting, mitochondrial genome 


\section{ABSTRACT}

Evolutionary rates and strength of selection differ markedly between haploid and diploid genomes. Any genes expressed in a haploid state will be directly exposed to selection, whereas alleles in a diploid state may be partially or fully masked by a homologous allele. This difference may shape key evolutionary processes including rate of adaptation and inbreeding depression, but also the evolution of sex chromosomes, heterochiasmy and stable sex ratio biases. All diploid organisms carry haploid genomes, most notably the haploid genomes in gametes produced by every sexually reproducing eukaryote.

25 Furthermore, haploid expression occurs in monoallelic expressed imprinted genes, in sex chromosomes and in organelles, such as mitochondria and plastids. A comparison of evolutionary rates among these haploid genomes reveals striking parallels. Evidence suggests that haploid selection has the potential to shape evolution in predominantly diploid organisms, and taking 30 advantage of the rapidly developing technologies, we are now in the position to quantify the importance of such selection on haploid genomes. 


\section{INTRODUCTION}

Ploidy is defined as the number of homologous chromosomes present in a cell and varies across taxa. The most commonly encountered states among organisms are haploidy (one copy of chromosomes) and diploidy (two copies of homologous chromosomes). The terms "haploid" and "diploid" were first coined in 1905 by the German botanist Eduard Strasburger in a publication describing the processes of meiotic divisions in several plant species (Strasburger 1905). Strasburger (1894) was also the first to recognise that the two alternating phases were an inevitable consequence of sexual reproduction in eukaryotes. The necessary alternation of diploid and haploid phases during sexual reproduction (Kirkpatrick 1994; Mable \& Otto 1998) allows for selection to act during both phases. The rates of evolution differ strikingly between a diploid and a haploid genome (see Section "Haploid Selection in Theory"), and taking both phases into account when studying diploid organisms will improve our understanding of evolution.

In addition to the haploid genomes found in gametes, predominantly "diploid" organisms contain a range of other haploid genomes (Figure 1) and studying these may help making more accurate predictions for evolutionary dynamics. The diversity of "haploid" genomes includes the genomes rendered functionally haploid through imprinting and targeted silencing of one allele, the haploid genomes of unmatched sex chromosomes in organisms with heterogametic sex determination and the haploid genomes of organelles such as mitochondria and plastids. The genetics of some of these haploid genomes are well studied, whereas others are still a conundrum and it may be worth zooming in on these to fully understand the role of haploid genomes in the evolution of "diploid" organisms.

60 While there is substantial body of theoretical work on the relative importance of selection occurring in haploid and diploid genomes, particularly in biphasic life cycles, the empirical evidence is scarce. Several reasons may explain the current lack of data for the impact of a biphasic life cycle on evolutionary processes. For one, much of our focus is directed towards diplontic 
organisms, which spend most of their life cycle as diploids and exhibit only a very short haploid gametic phase. Furthermore, technical limitations may have contributed to the current scarcity of empirical evidence for the impact of haploid selection in diplontic organisms. This may be the right time to revisit the topic and improve our understanding by taking novel approaches made possible with the advent of ever improving sequencing and other molecular technologies (see also Box 1) and by expanding our investigations across a wider range of taxonomic groups.

\section{HAPLOID SELECTION IN THEORY (500 WORDS)}

75 Selection acting on haploid and diploid genomes in the same organism changes the evolutionary dynamics in a number of ways. First of all, alleles expressed under haploidy will be directly exposed to selection whereas alleles expressed in a diploid genome may be partially or completely masked by dominance and hence escape selection (Crow \& Kimura 1970). In addition, the mutation rate in a diploid genome is generally assumed to be higher than in a haploid genome because of the higher number of copies present in a population. These differences between haploid and diploid genomes in turn may cause variation in expression and result in genomic conflicts, particularly if these differences in ploidy coincide with differences in the expression

85 context such as different tissues, life stages and/or sexes. The potential evolutionary consequences resulting from the co-existence of haploid and diploid genomes have been extensively assessed in theoretical studies with an overview of these provided here below.

90 The masking effect in diploid genomes is a key difference to haploid genomes and may affect evolution and the underlying change in allele frequencies in two fundamental aspects: (i) positive selection favouring beneficial alleles may be hampered in a diploid genome if these are not completely dominant (Orr \& Otto 1994) and (ii) purifying selection will be less efficient in removing recessive deleterious alleles in a diploid genome (Crow \& Kimura 1970). The difference in the efficiency of positive selection due to ploidy may directly affects the rate of adaptation and often accelerates the spread of beneficial de 
novo mutations in haploid populations compared to diploid populations (Orr \& Otto 1994). Similarly, the effective removal of deleterious mutations has potentially important implications for the genetic load in a population (Charlesworth \& Charlesworth 1992), which is of particular interest in the case of inbreeding depression (Charlesworth \& Charlesworth 1987; Losdat et al. 2014). In fact, even a short window of haploid selection may effectively remove deleterious mutations from a population and thereby reduce the genetic load (Otto et al. 2015). The efficiency of haploid selection described here has far-reaching consequences and may affect the evolution of diplontic organisms in many ways.

The assumption of an increased mutation rate in diploid genomes is largely

110 based on the fact that a diploid genome contains double the number of nucleotides compared to a haploid genome. In fact, the doubled mutation rate in diploid genomes is an underlying assumption of many theoretical models investigating the evolution of biphasic life cycles and ploidy (e.g. Crow \& Kimura 1965; Kondrashov \& Crow 1991). However, a recent comparison of

115 the rate and type of mutation occurring in haploid and diploid yeast strains showed that the difference in mutation rates between the two is more complex than previously assumed (Sharp et al. 2018). In fact, haploid strains seemed more susceptible to single-nucleotide mutations suggesting that the assumption about mutation rates in diploid and haploid genomes may need careful revisiting

Variation in ploidy of genomic regions, chromosomes or even entire genomes is often discussed in the context of genomic conflict, particularly if alleles are exposed to differential of antagonistic selection. Sexually antagonistic

125 selection and the potential for intra-locus sexual conflict may lead to the targeted silencing through imprinting of one allele in a specific sex, (e.g. Arnqvist \& Rowe 2005; Day \& Bonduriansky 2004) rendering this locus functionally haploid in this sex. Similarly, genetic sex determination systems often involve ploidy differences between males and females either for sex 130 chromosomes or the entire genome (Bachtrog et al. 2014). Such ploidy differences between the sexes can be driven by sexually antagonistic 
selection (Immler \& Otto 2014). However, whether sex differences in ploidy are causing such conflicts or whether they may help mitigating existing conflicts is not entirely clear, and the answer may vary for the different scenarios.

Biphasic life cycles with alternating diploid and haploid phases offer an additional opportunity for genomic conflict between the phases even in predominantly diploid organisms (Immler and Otto 2018). One of the first to specifically consider selection at the haploid gametic phase was Haldane (1924) who showed that selection at this level is particularly effective compared to selection at the diploid stage. Haldane's model was the basis for deterministic and stochastic models showing that alternating phases of haploid and diploid selection (Scudo 1967; Hartl 1977) and antagonistic

145 selection across the ploidy levels and between the sexes (Ewing 1977; Immler et al. 2012) can lead to the maintenance of stable genetic polymorphisms. The potential conflict between the diploid gamete producing organism and its haploid gametes arising from such antagonistic selection has specifically been discussed in the context of sperm competition. A general prediction is that such a conflict should lead to the silencing of the haploid gametic genome to reduce the risk of a conflict (Haig and Bergstrom 1995). The reason for this prediction is a potential conflict of interest over controlling the sperm phenotype and hence the ability to successfully outcompete potential rival sperm. Two theoretical studies, one assuming diploid male control (Parker 1993) and one assuming haploid gametic control (Parker and Begon 1993) over the evolution of sperm traits directly related to their competitive ability showed that the potential conflict between the diploid male and its haploid sperm over ejaculate expenditure strongly affect the Evolutionary Stable Strategies of male reproductive investment. In contrast, a more recent theoretical study predicted that the fierce competition among sperm within the ejaculate of a male in fact may increase the rate of haploid expressed genes due to an increased fixation rate of advantageous alleles (Ezawa \& Innan 2013). Furthermore, the benefits of purifying selection may favour the evolution of haploid selection, particularly when alleles are under ploidally 165 antagonistic selection (Otto et al. 2015). 
The role of haploid selection in combination with sexually antagonistic selection may affect a range of evolutionary processes. Sex-specific haploid selection in combination with negative epistasis between two loci under sexually antagonistic selection is strong enough to drive sex differences in recombination rate due to a reduced recombination in the sex with the strongest value of epistasis (heterochiasmy; Lenormand 2003). In fact, heterochiasmy is predicted to evolve even in the absence of epistasis if haploid selection on alleles differs between males and females and if the two loci are in linkage disequilibrium because of some other mechanism. In addition, sex-specific selection on haploid gametes may also predict reduced recombination rates on sex chromosomes and an enrichment for haploid expressed genes on the sex chromosomes (Scott \& Otto 2017). Furthermore, haploid selection can drive transitions between male and female

180 heterogametic sex determination systems even if the linkage between the sex determining locus and the sexually antagonistic locus are not tightly linked, a strict requirement for such transition with loci under purely diploid selection (Scott et al. 2018b). Such transitions may affect population sex ratios, which may increase or decrease with the spread of a new sex chromosome

185 suggesting that new sex chromosomes may evolve without selection for balancing sex ratio. In addition, purifying selection on male haploid gametes may explain stable sex ratio distortion as a result of the balance between the advantages of purging deleterious mutations via haploid selection, and the disadvantages of haploid selection on the sex ratio (Hough et al. 2013).

\section{GAMETIC HAPLOID SELECTION - EMPIRICAL EVIDENCE}

Although the potential importance of haploid selection for evolutionary - and more broadly biological - processes is indisputable as shown by the theoretical work described in the previous section, empirical studies investigating haploid selection are still scarce. At this stage, it is important to distinguish between male and female gametes. Already Haldane (1924) noted that the scope for selection in female gametes may be much smaller than in male gametes, because a majority of mature eggs/ovules produced by a 
200 female stand a high chance to be fertilised, whereas a tiny fraction of male gametes will end up fertilising an egg/ovule. I will therefore discuss the potential for haploid selection in the two sexes separately. In addition, our understanding of haploid gametic selection differs significantly between plants and animals. Haploid gametic selection is widely accepted in plants, in fact so

205 much so that it has found its applications in agricultural practices and crop breeding (e.g. Zamir \& Vallejos 1983; Clarke et al. 2004; Domínguez et al. 2005). The reason for this is that mitotic cell division and growth is necessary during pollen tube growth leading to the expression of up to $60 \%$ of genes in haploid pollen (Haldane 1932; Mascarenhas 1990; Walbot \& Evans 2003;

210 Borg et al. 2009) of which about 10\% are expressed exclusively in the haploid stage (Honys \& Twell 2004; Borg et al. 2009; Arunkumar et al. 2013).

In contrast, a persistent view holds that the scope for haploid selection in animals is minimal. The sperm phenotype and its ability to fertilise an egg

215 determine the fitness of the diploid male, and particularly males facing sperm competition are selected to produce competitive sperm phenotypes (Birkhead and Moller 1998). As mentioned above, due to the potential conflict arising from differential selection in haploid gametes and diploid organisms, a general assumption is that the diploid male has control over sperm phenotypes and

220 the haploid sperm phenotype has a minimal impact on the sperm phenotype (REF). This view was confirmed early on by empirical findings that Drosophila and mice mutant males producing sperm that lacked a nucleus were still capable of fertilising an egg (Muller \& Settles; Lindsley \& Grell 1969; Lyon et al. 1972). In addition, even those genes expressed in early haploid spermatids 225 appeared to be shared through cytoplasmic bridges rendering the spermatids functionally diploid (e.g. Dym \& Fawcett 1971; Caldwell \& Handel 1991). A statement in the foreword to the Proceedings of the conference on Gamete Selection in Plants and Animals in 1975 saying "haploid transcription is apparently a relatively rare and insignificant phenomenon among higher

230 animals" (Mulcahy 1975) epitomised this general idea that there is little opportunity for haploid selection to occur. However, an increasing body of empirical evidence is continuously challenging and overturning this view. 


\section{Haploid gene expression}

235 Active transcription of genes at the haploid post-meiotic stages is a key prerequisite for haploid gametic selection to occur. As mentioned above in plants, haploid gene expression and hence selection during pollen tube growth is extensive (e.g. Mascarenhas 1990; Sari-Gorla \& Frova 1997). In animals, evidence for postmeiotic gene expression is more complicated to

240 obtain but more recent studies of post-meiotic transcription have since revised the prevailing view and post-meiotic gene expression has been reported in several taxa including Drosophila and a range of mammals (reviewed in Erickson 1990; Hecht 1998; Steger 1999; Kanippayoor et al. 2013).

245 A possible explanation for the fertilisation ability of genome-depleted sperm in earlier experiments is the syncytial organisation of early spermatids, where several (over one hundred in mammals and 64 in $D$. melanogaster) haploid cells stay connected by cytoplasmic bridges allowing the distribution of transcripts (Erickson 1973). This characteristic suggests that the sharing of nascent RNAs among early spermatids renders these cells functionally diploid. While evidence for sharing is strong for some genes such as the allelic expressed protamines $h$ Prot 1 and $h$ Prot 2 in humans and similar alleles in mice, the sharing is not always perfect and expression biases may persist (Kanippayoor et al. 2013). The lack of sharing and the compartmentalisation

255 and immediate translation of mRNA of the house mouse gene spam1, for example, supports the idea of haploid expression of this gene associated with male infertility (Zheng et al. 2001). Previous estimates suggested that up to several hundred genes are expressed at the post-meiotic genes in several taxa (Joseph \& Kirkpatrick 2004). An important next step is to take a broader

260 approach and create transcriptome maps of nascent RNAs in post-meiotic spermatid cells to identify all allelic expressed genes.

The transcriptional and translational activity in mature sperm is another much debated topic (Ren et al. 2017). The first evidence for active translation in

265 mature sperm came from bovine sperm that were observed to incorporate radio-labelled ribonucleoside triphosphates into RNA and protein molecules (MacLaughlin \& Terner 1973; Premkumar \& Bhargava 1972). The general 
thought, however, was that this translational activity was confined to the sperm mitochondria (Miller \& Ostermeier 2006) until a more recent study demonstrated translational activity in mature sperm of several mammalian species and showed that mammalian sperm translate nuclear-encoded proteins by mitochondrial-type ribosomes (Gur \& Breitbart 2006; Gur \& Breitbart 2008). Such translational activity in late spermiogenesis and mature sperm might also explain the striking finding of eight proteins differing in abundance in X- and Y-bearing bull sperm (Scott et al. 2018a).

Patterns of transcriptional and translational profiles in the haploid post-meiotic phases of spermiogenesis are likely to vary markedly across species. Spermatogenesis exhibits remarkable variation across taxonomic groups in 280 the way germ cells are organised and divide before, during and after meiosis, the genes that are involved and most likely also the transcriptional and translation activity in post-meiotic spermatids and mature sperm (Ramm et al. 2014). A key characteristic with potential relevance to our understanding of haploid selection that varies across species is the way the sperm nucleus is condensed and how histones are replaced during sperm maturation: histones are retained to varying degrees ranging from an estimated $1 \%$ in human sperm, 10 to $50 \%$ in house mouse, and up to $100 \%$ in the zebrafish. The specific location and nature of different histone variants may provide further information about the functional activity of sperm nuclei and how it may vary across species.

Furthermore, the condensation of the sperm nucleus occurs in a highly organised manner, exhibiting a distinct hairpin-structure with DNA loops similar to the chromosome structure in condensed mitotic cells (Ward 2018).

295 Such conserved organisation results in specific genome regions to consistently being located on the surface of the nucleus, whereas other regions are hidden within the nucleus and may be inaccessible to any transcription and/or translation factors. Whether the specific location within the nucleus has any significance for the activity of genes is currently speculative 300 at best, but certainly deserves further attention. 


\section{Genetic diversity and genotype-phenotype links among male gametes}

The processes of segregation and recombination occurring during most meiotic processes of spermatogenesis lead to the prediction of substantial genetic variation among sperm within an ejaculate. The number of chromosomes determines the variation among sperm due to segregation and the rate of male recombination adds anadditional level of variation. The latter may be of particular importance if genes under haploid selection show any additive effects or signs of epistasis. Cohen $(1967,1973)$ reported a positive association between the recombination rate (chiasma rate) and the number of sperm produced across a range species. Cohen's interpretation of this finding was that recombination events may lead to the production of suboptimal sperm and in order to compensate for the number of suboptimal sperm, males produce relatively more sperm. Data on recombination events are now

315 available at a higher resolution, and his observation can be tested on a larger sample size. More generally, single-sperm genotyping may be a great tool to assess genetic variation among sperm within a male's ejaculate and get a better estimate of the recombination rate and its role in generating genetic variation among sperm (e.g. Lu et al. 2012; Wang et al. 2012; Xu et al. 2015; see also Box 1).

A logical next question is then: What is the evidence for the role of this genetic variation to contribute to the phenotypic diversity among sperm within an ejaculate? A study in the zebrafish reported allelic divergence across the entire genome between sperm pools selected for different longevity phenotypes from the ejaculate of individual males (Alavioon et al. 2017). Similarly, in the Astyanax cavefish, sperm carrying specific alleles exhibited a different reaction phenotype in response to exposure to Hoechst dye (Borowsky et al. 2018). Moreover, X- and Y- carrying bull sperm differ in eight

330 mitochondrial membrane proteins, ranging from cytoskeleton-protein coding genes to NADH dehydrogenases involved in ATP production via oxidative phosphorylation and acetyl-CoA carboxylase, both of which play key roles in energy production (Scott et al. 2018a). All these very recent findings support the idea that the haploid genome does play a role in determining sperm 335 phenotypes, but which genes exactly are involved needs further investigation. 
A first tentative conclusion we may draw here is that phenotypic traits affected by the haploid genome generally seem to be functional phenotypes rather than morphological structures.

340 The genetic diversity among sperm is certainly not the only mechanism affecting phenotypic variation among sperm of one male. Even in the mitotically-produced, genetically-identical sperm of haploid males in eusocial insects, morphological variation is substantial suggesting that the phenotypic variation in size is largely determined by variation during spermatogenesis

345 rather than affected by the sperm haplotype (Fitzpatrick \& Baer 2011). Similarly, an elegant experiment performing crosses between Drosophila lines selected for long and short sperm suggested that the haploid sperm genome has little influence on the length of sperm as no distinct size classes were found among the male F1 crosses (Pitnick et al. 2009). These results support

350 the idea that at least morphological sperm traits are likely to be under the control of the diploid male and reflect variation in male condition (Holt \& Van Look 2004). Add something more general here in response to Miake's comment

\section{Evidence for impact of haploid gametic selection on offspring} phenotypes

Gametes from one male are expected to compete (Manning \& Chamberlain 1994; Haig and Bergstrom), and some gametes may have an advantage at fertilising eggs over their sibling gametes. In plants, competition among pollen

360 of different males and also within males have been shown to not only affect fertilisation success rates but also directly improve offspring fitness (e.g. Snow \& Spira 1996; Aronen et al. 2002; Lankinen et al. 2009). In addition, within plant pollen competition appears to reduce inbreeding depression in Dalechampia scandens (Armbruster \& Gobeille Rogers 2004). Finally,

365 selection on pollen tube growth occurring during fertilisation has enabled cropbreeders to obtain cold-tolerant chickpea Cicer arietinum and tomato Solanum lycopersicum plants in the next generation (Clarke et al. 2004; Domínguez et al. 2005). In contrast, evidence for competition and selection among haploid sperm affecting offspring fitness is less abundant. 
Evidence for a role of selection on sperm genotypes has been reported in the house mouse Mus musculus where sperm reaching the fallopian tube showed lower levels of DNA fragmentation (Hourcade et al. 2010) and in boar, where chromatin unstable sperm were less likely to reach oocytes in vivo (Ardon et 375 al. 2008). These two examples suggest that selection on the ability of sperm to reach the site of fertilisation is at least associated with the genetic quality of the sperm and prevent suboptimal sperm from fertilising an egg. The most direct evidence for a link between the sperm phenotype and the sperm genotype comes from two recent studies in fish. In the Mexican cavefish Astyanax, sperm haplotypes seem to be directly associated with phenotypic variance, where sperm from a hybrid male could be distinguished into two phenotypic groups (Borowksy et al. 2018). Similarly, a study in the zebrafish Danio rerio showed not only a direct link between sperm phenotypes and offspring fitness from early live into adulthood, but also a link between the

385 haploid sperm genotype and the selected sperm phenotype (Alavioon et al. 2017). Signs of allelic divergence between two sperm pools selected for differential phenotypes from within the same ejaculate were found across the entire genome, although some of these signals may be false positives due to the effects of linkage and background selection. A better understanding of the

390 expected genetic variation and the role of linkage in determining genetic variation among sperm (and gametes more genrally) is needed to improve the accuracy of such analyses.

\section{Haploid selection in female gametes}

395 As mentioned above the opportunity among female gametes generally is thought to be much more limited for three reasons, namely the costs of female gamete production, the high fertilisation success in female gametes and the delayed completion of the second meiotic division shortly before, during or after fertilisation (Immler \& Otto 2018). Nevertheless, there are several instances where selection among female gametes may still be of importance and are therefore worth further consideration. 
A possible opportunity for haploid selection on female gametes occurs precisely during fertilisation and the completion of the second meiotic division.

405 Arresting the second meiotic division in the metaphase until a sperm enters and egg provides an opportunity for the female to assess the genetic match between the paternal and the maternal genomes forming the diploid zygote. Direct empirical studies of such processes are currently lacking, but an interaction between male and female haploid pro-nuclei has been suggested

410 in the context of several findings. The perhaps most striking example of a possible process of "choice" at the haploid level comes from a marine invertebrate, the comb jelly Beroe ovata, where polyspermy leads to the presence of several sperm pronuclei in one egg and the female pronucleus has been observed to migrate between these (Carré \& Sardet 1984); for a

415 video clip of the striking process see:

https://jellybiologist.com/2013/05/20/video-can-an-egg-choose-the-sperm-itlikes-best/). Physiological polyspermy - as opposed to pathological polyspermy, which is detrimental to the development of the zygote - is widespread across taxa and in many cases appears to be obligatory for successful

420 fertilisation and development of an egg (see Snook et al. 2011 for review). However, the reason for physiological polyspermy is unclear and the scope for a possible "mate choice" process occurring at this stage warrants further careful investigation.

425 Direct empirical tests for possible evidence of assortative fusion based on the haploid gametes are scarce and come exclusively from animals. Two studies in whitefish Coregonus spp. and Atlantic salmon found no evidence for a assortative fusion with respect to different MHC alleles (Wedekind et al. 2004; Promerová et al. 2017), whereas a study in the three-spined stickleback

430 Gasterosteus acculeatus reports a possible role of assortative fusion to finetune MHC genotypes in resulting offspring (Lenz et al. 2018). Similarly, in house mice Mus musculus a possible interaction between the male and the female haploid genomes have been suggested to explain non-mendelian inheritance of certain alleles (Wedekind et al. 1996; Nadeau 2017). Nadeau

435 (2017) suggested that these haploid genome interactions could be conditiondependent. Such condition dependence more generally would further explain 
the difficulty to provide firm and clear evidence for haploid selection to occur. However, at this stage this idea is still rather speculative and needs careful further testing.

\section{Evidence for roles of haploid selection in evolutionary processes}

Gametic haploid selection has been suggested to play a potentially important role in a range of evolutionary processes including adaptation, purifying selection, the evolution of sex chromosomes and heterochiasmy (see section

445 "Haploid Selection in Theory"). Haploid gametic selection was discussed as a possible explanation for a lack of sex-bias in expression of mito-nuclear genes across several species due to the potentially tight interaction between the sperm and its mitochondria (Dean et al. 2014). The reasoning was that individual sperm can only carry either an $\mathrm{X}$ or $\mathrm{Y}$ chromosome and haploid 450 expression of mitonuclear genes would select against sex linkage, as such linkage would render $50 \%$ of sperm non-functional. More recently, the role of haploid gametic selection in the evolution of sex chromosomes was demonstrated in two Rumex species (Sandler et al. 2018). By examining the evolution of gene expression in flower buds and pollen to test for signatures of

455 haploid selection acting during plant sex chromosome evolution, the authors found a bias of genes with high ancestral pollen expression bias on sex chromosomes compared to autosomes. Furthermore, genes on the $Y$ chromosome were more likely to become enriched for pollen expressed genes with a significant loss of genes with low pollen expression levels.

460 Haploid selection was also thought to be the most likely explanation for the observed variation of heterochiasmy across a range of plant species (Lenormand \& Dutheil 2005). Similarly, it seemed the most plausible explanation for observed heterochiasmy in a coral Acropora millepora and may also explain a low polymorphism level of one linkage group in the male 465 (Wang et al. 2009). In this study, the male genotype was inferred from his sperm sample, and haploid selection may result in a difference at some loci between the ejaculate genotype and the genotype in the adult male. This may occur for example if one homologous chromosome corresponding to one linkage group produces functional sperm, while the other chromosome may 
carry deleterious alleles resulting in non-functional sperm. These ideas are untested at this moment, but they deserve further attention.

All the evidence for gametic haploid selection discussed in the sections above is focusing on the possible mechanisms allowing for genes to be expressed in

475 haploid post-meiotic cells, evidence that haploid genotypes affect pollen and sperm phenotypes and how such phenotypic variation may affect offspring phenotypes. An important additional and more recent approach to look for signs of haploid selection is to take advantage of novel sequencing technologies to estimate the strength and nature of selection occurring across

480 the genome (Box 1). By using a combination of quantitative genetics, population genetics and genomics it will be more than ever possible to assess the true potential for haploid selection in animal male gametes.

\section{OTHER HAPLOID GENOMES IN “DIPLOID” ORGANISMS}

485 Besides the haploid gametic phase in sexually reproducing eukaryotes, diploid organisms carry a range of other haploid genomes and genes. Although by definition "diploid" organisms are carrying two sets of alleles in most cells and biallelic gene expression is generally the norm, haploid allelic expression of genes can be found at many different levels. Monoallelic

490 expression may be found in imprinted genes, genes located on sex chromosomes and on chromosomes in haplo-diploid species. Furthermore, eukaryotic cells rely on the haploid genomes of organelles such as mitochondria or plastids). Studying the evolutionary dynamics in each of these different scenarios may enable identify possible parallels evolutionary patterns among the different systems (summarised in Table 1).

\section{Haploidy through imprinting}

Genetic imprinting and gene silencing may render individual loci or entire genomic regions or chromosomes effectively haploid. Random monoallelic expression exists at the genome-wide scale and affects a large number of coding genes (Chess 2012). Random monoallelic imprinting is expected to slow down purifying selection compared to alleles with complete dominance 
but may increase the rate of positive selection compared to loci with biallelic expression. Similar to random monoallelic expression, sex-specific imprinting consistently silences loci depending on their parental origin (Reik \& Walter 2001). Sex-specific imprinting has been extensively discussed particularly in the context of sexual conflict over gene expression between the paternal and the maternal genome in zygotes (e.g. Haig 2000). The gene MEDEA is essential for seed development and is a maternally imprinted gene with a silenced paternal allele resulting in haploid expression. As expected for haploid expressed alleles, MEDEA shows signs of accelerated selection in the outcrossing species Arabidopsis lyrata (Spillane et al. 2007). Sex-specific expression and random monoallelic expression are likely to show similar patterns of purifying and positive selection, if sex-specific imprinting varies

515 across individuals.

Imprinting is also a mechanism for dosage compensation to account for the ploidy differences in the sex chromosomes ( $\mathrm{X}$ or $\mathrm{Z}$ ) between the homogametic and heterogametic sexes. In mammals for example, dosage compensation is achieved by the random inactivation of one of the two $X$ chromosomes in females through DNA methylation, histone modification and large-scale chromatin re-structuring (Brockdorff \& Turner 2015). Similar to the observations made for randomly silenced loci described above, purifying selection in inactivated genes on the $X$ chromosome is reduced compared to X-linked genes that escape inactivation (Park et al. 2010). The reason for this is that random inactivation allows deleterious alleles to hide from selection. Interestingly, however, positive selection does not seem to differ between the inactivated loci and loci escaping inactivation in X-linked genes.

\section{Haploid chromosomes and genomes}

Heterogametic sex determination renders one of the two sex chromosomes consistently haploid ( $Y$ and $W$ ) and the other one ( $X$ and $Z$ ) haploid for $1 / 3$ of the time in the heterogametic sex. Concentrating entirely on the ploidy state of each sex chromosome, we would expect purifying selection to be enhanced on $\mathrm{Y}$ and $\mathrm{W}$ compared to $\mathrm{X}$ and $\mathrm{Z}$ chromosomes. However, these predictions 
are strongly confounded by the fact that heterogametic sex chromosomes evolve asexually and hence recombination as an efficient means to remove deleterious mutations from chromosomes does not occur. Furthermore, factors such as reduced effective population size (Charlesworth 1978; Gordo

540 \& Charlesworth 2000) and background selection (Charlesworth 1996) should further contribute to the degeneration of the heterogametic sex chromosome making predictions for the $\mathrm{Y}$ chromosome evolution challenging. Nevertheless, genome sequencing data from 11 genes in nine Drosophila species supports the idea of recurrent events of positive selection and 545 uncompromised purifying selection against strongly deleterious mutations (Singh et al. 2014). Only mildly deleterious mutations appear to be maintained by background selection.

$X$ and $Z$ chromosomes exist in a haploid state in $1 / 3$ of their evolutionary time 550 either in company of a heterogametic non-recombining $Y$ or $W$ or without any homologous chromosome in species with X0 sex determination systems. Genes expressed during this time are again predicted to experience enhanced levels of purifying and positive selection compared to diploid autosomes. These predictions are confirmed in the sequencing data from 12

555 Drosophila species, where elevated biased codon usage in genes on the $X$ chromosomes supports the hypothesis of increased purifying selection, whereas increased substitution rates as a sign of positive selection were only observed in some lineages (Singh et al. 2008).

560 The selection dynamics described above for sex chromosomes apply also to species with haplo-diploid sex determination (haploid males and diploid females). However, any genes exclusively expressed in males will only experience haploid selection, and genes expressed exclusively in females only diploid selection. The low effective population sizes characteristic for 565 eusocial haplo-diploid insects may be a confounding factor when estimating strength of selection (Romiguier et al. 2014). Nevertheless, haploid males in eusocial insects may be the equivalent of the haploid gametes produced by a diploid male. Studying the genomic signatures of selection in eusocial insects 
may therefore reveal important information about what we would expect in one fertilisation event between a male and a female.

\section{Haploid organelles}

The haploid genomes of organelles typically found in eukaryote organisms provide an additional opportunity to understand the genomic evolution in

575 response to haploid selection. Organelle genes as those found in mitochondria and plastids have been regularly used for phylogenetic reconstructions across taxa under the erroneous assumption, that these genes may serve as neutral markers due to a lack of selection. However, this view has since been changed and the fundamental differences between nuclear and organelle genes have been increasingly recognised (Ballard \& Whitlock 2004; de Vries \& Archibald 2018). The evolution of mitochondria and plastids may be assessed at two levels, one at the individual organelle level and one at the organismal level of the individual carrying the organelle in its eukaryotic cells. The seemingly rapid evolution of mitochondrial genes for example may find an explanation partially in the nature of its haploid genome on one hand and in the vast population sizes on the other hand. Each eukaryotic cell may contain up to 2000 mitochondria and these numbers duplicate during each mitotic division. Such vast numbers are expected to show rapid rates of evolution even in asexually reproducing populations,

590 particularly given that positive selection and purifying selection are expected to be strong in these haploid genomes.

However, estimates of mitochondrial evolution are generally performed at the organismal level. This approach may not be a due reflection of the processes

595 affecting the evolution of mitochondria (and other organelle) genomes.

Recombination rates in mitochondria vary markedly and may range from nonrecombinant in mammals to relatively high rates of recombination in some species of yeast and in species with bi-parental mitochondrial inheritance (Barr et al. 2005). Mitochondria within an individual are rarely homogeneous

600 but in effect exhibit substantial genetic variation and bottlenecks as those assumed to occur during inheritance are likely to be nowhere as severe as 
assumed, as a single oocyte may carry over as many as $10^{6}$ mitochondria (Shoubridge \& Wai 2007; Radzvilavicius et al. 2016). In addition, (deleterious) mitochondrial mutations only have a noticeable effect on organismal fitness at frequencies as high as 60\%-80\% (Rossignol et al. 2003). Evolutionary constraints affecting mitochondrial evolution may be caused by the tight mitonuclear interactions. Estimates of purifying selection any directional selection as those reported for example in mammals may therefore not directly reflect the population genetic processes occurring at the mitochondrial level.

610 However, they will still be important to understand large-scale evolutionary processes. Given the complexity of organelle biology, more in-depth investigations are required to fully understand the genetic and genomic dynamics explaining the evolutionary pathways described across at the macro-evolutionary level.

615

\section{CONCLUSIONS AND FUTURE DIRECTIONS}

While absolute values of strength of selection and rate of evolution vary across the studies investigating the role of haploid expression for purifying and positive selection, the qualitative patterns emerging from across the

620 different haploid genomes (Table 1 ) are surprisingly similar. The role of haploid selection for purifying and positive selection finds strong support in many of the scenarios discussed above including in haploid gametic selection, imprinted genes and haploid selection sex chromosomes. The complex biology and the multiple levels of selection experienced by organelles, renders the qualitative and quantitative description of evolutionary patterns more challenging. However, research is heading in exciting directions and intriguing new data should soon be available to answer the questions raised in the previous sections.

630 This review clearly demonstrates that the effects of haploid selection at all levels may have striking effects for evolutionary processes in diploid organisms. In particular, haploid gametic selection may be more important in animals (and plants) than assumed so far. In order to obtain a complete picture, questions about the mechanisms maintaining genetic variation at loci 
under haploid selection are among the most important to be addressed. One possibility is that antagonistic selection across the ploidy levels and/or the sexes maintains a stable polymorphism. Balancing selection is non-mutually exclusive alternative mechanism maintaining genetic variation at loci under haploid selection. Scenarios of balancing selection may be found in changing conditions during fertilisation events affecting gene expression and metabolic rates in both, pollen and sperm. This leads to the next question about the nature of the genes expressed during the haploid phase. In plants, these are generally housekeeping genes (Arunkumar et al. 2014), whereas in animals such information is currently missing. Additive genetic variation and epistasis among such genes may result in distinct sperm cohorts, where more than one allele combination can be optimal. In addition, soft selection among gametes produced by one individual may be an additional factor that helps maintaining genetic variation in a population. However, these ideas are currently untested both, in plants and animals.

Overall, understanding the evolutionary dynamics in the different haploid genomes described here will substantially contribute to our understanding of evolutionary processes as a whole. The identification of the importance of purifying and directional selection at the haploid gametic stages for example

655 may help provide the information needed to link apparent discrepancies for mutation rate estimates based on phylogenetic datasets compared to those at the individual and family level. It may also provide explanations for the maintenance of genetic variation despite apparently strong selection on the diploid organisms and may explain why the signs of inbreeding depression

660 are often not as severe as expected even in small populations of endangered organisms.

\section{ACKNOWLEDGEMENTS}

I would like to thank Mike Whitlock for giving me the opportunity to write this review and for his invaluable feedback. I am particularly grateful to Glyn McAlister and the Norfolk Fire and Rescue Service for preventing our house from burning down while writing this article. SI is funded by grants from the European Research Council (HapSelA-336633), the Knut and Alice 
Wallenberg Foundation (Wallenberg Academy Fellowship) and the Human 670 Frontier Science Program (HFSP). 
Box 1: Genomic signatures of haploid gametic selection

675 With the advent of continuously improving sequencing technologies, we may take entirely new approaches to assess and understand the importance of selection on haploid genomes in diploid organisms. Whole genome sequencing is a powerful tool to study rates of (i) mutation, (ii) recombination and (iii) selection - all key ingredients for understanding population ecology 680 and evolution.

(i) Our estimates of mutation rate are continuously improving with long-read sequencing technologies that allow the identification of true de novo mutations compared to technical faults due to sequencing errors or to bioinformatic 685 mistakes (improved by increased read-length technologies). With the help of single-cell sequencing we can now assess mutation rates at the level of gametes and obtain estimates of purifying selection by comparing mutation rates in sperm to mutation rates estimated in pedigrees.

690 (ii) Similarly, estimates of recombination rates may also benefit from singlecell sequencing and will improve our understanding of the genetic variation present among sperm due to segregation and recombination. Recombination estimates from pedigrees are likely to underestimate the frequency of recombination events, particularly if these events have deleterious effects due disrupted allele combinations.

(iii) Sequencing technologies also enable us to identify signs of selection. Pioneering studies in the outcrossing plants species Capsella grandilfora and Arabidopsis thaliana provide invaluable insights into what we may expect to 700 find in genes exclusively expressed during the haploid pollen phase, for genes expressed in both phases and in genes expressed purely in the diploid phase (Arunkumar et al. 2013; Gossmann et al. 2014). In both studies, haploidexclusive genes had more sites under strong purifying selection, a greater proportion of adaptive substitutions, and faster protein evolution compared to 705 genes exclusively expressed in the diploid phase. The effect of purifying selection against strongly deleterious mutations persisted also in genes 
expressed during both phases, whereas the signs of directional selection were only marginally higher in genes with bi-phasic expression compared to diploid-exclusive genes.

Based on these important insights from plants it may be worth looking at rates of mutation and selection in animals and compare genes expressed in haploid spermatids to those expressed exclusively in the diploid organism. In fact, a study on human populations reported a surprisingly high signal of purifying selection in such spermatid-expressed genes and the authors state: "Although there is no guarantee that gametic selection is beneficial to the organism, if significant purging can occur during gametogenesis (even at the diploid germcell stage), selection at this phase can dramatically enrich the proportion of 'purified' genomes for fertilization” (Reed \& Aquadro 2006). Furthermore, genes involved in the condensation of spermatogenic DNA expressed in haploid spermatids are often rapidly evolving between mammalian species (Good \& Nachman 2005) and more generally, rates of protein evolution have been found to be positively correlated with developmental timing of expression of genes involved in spermatogenesis (Good \& Nachman 2005;

725 Podlaha \& Zhang 2003; Podlaha et al. 2005). A striking example of extremely high levels of insertion-deletion variation of an alanine-rich repetitive motif in natural populations of Mus domesticus and M. musculus was found at Testisspecific gene a8 (Tsga8), a spermatogenesis-specific gene expressed during postmeiotic chromatin condensation and nuclear transformation (Good et al. 2011).

Whether haploid expression is also (partly) responsible for the rapid evolution in reproductive genes more generally (Swanson \& Vacquier 2002) needs further investigation. The fact that $\mathrm{dN} / \mathrm{dS}$ exceeds 1 for genes whose products 735 are found in mature sperm (Ezawa \& Innan 2013) combined with the positive correlation between high dN/dS values expression during the post-meiotic haploid phases (Good \& Nachman 2005) and the X/Y-sperm specific protein phenotypes (Scott et al. 2018a) may indicate a possible role for haploid selection. Combining sequencing technologies with targeted experimental 740 crossing and experimental evolution are likely to provide exciting new insights. 
Figure 1. Illustration of the haploid genomes present in a "diploid" organism. (1) haploid male gametes (pollen and sperm), (2) imprinted genome regions or inactivated sex chromosome with haploid expression (3) haploid genomes 745 in organelles (mitochondria/plastids), (4) haploid sex chromosomes in the heterogametic sex, (6) haploid female gamete (eggs and ovules). 
Table 1. Summary empirical evidence for haploid gene expression and possible consequences such as elevated levels of purifying selection, positive selection and direct fitness effects of such haploid selection. For male gametes, the first indication is for evidence in plants and the second for evidence in animals. Evolutionary rates of male-specific genes in haplo-diploid organisms are comparable to those for unpaired sex chromosomes and

755 paired chromosomes in the heterogametic sex in the absence of inactivation. In contrast, genes under random imprinting on autosomes and sex chromosomes do not show an increase in purifying selection. The column "Fitness effects" refers to positive effects on organismal fitness as a result of purifying and/or positive selection on the haploid genome. Evidence in mitochondria and plastids is here focusing on signs of selection at the mitochondrial level (i.e. among individual mitochondria) rather than at the organismal level.

\begin{tabular}{|c|c|c|c|c|}
\hline Genome & $\begin{array}{l}\text { Haploid } \\
\text { expression }\end{array}$ & $\begin{array}{l}\text { Purifying } \\
\text { selection }\end{array}$ & $\begin{array}{l}\text { Positive } \\
\text { selection }\end{array}$ & $\begin{array}{l}\text { Fitness } \\
\text { effects }\end{array}$ \\
\hline Male gamete & Yes/Yes & Yes/Unknown & Yes/Yes? & Yes/Yes? \\
\hline Female gamete & Unknown & Unknown & Unknown & Unknown/Yes? \\
\hline $\begin{array}{l}\text { Paired sex } \\
\text { chromosome with } \\
\text { inactivation }(X)\end{array}$ & Yes & No & No & Yes? \\
\hline $\begin{array}{l}\text { Paired sex } \\
\text { chromosome in } \\
\text { heterogametic sex } \\
(X, Z)\end{array}$ & Yes & Yes & Yes & Yes \\
\hline $\begin{array}{l}\text { Unpaired sex } \\
\text { chromosome (Y, } \\
\text { W) }\end{array}$ & Yes & Yes & Yes & Yes \\
\hline $\begin{array}{l}\text { Randomly } \\
\text { imprinted genomic } \\
\text { regions }\end{array}$ & Yes & No & Yes & Yes \\
\hline Organelle genomes & Yes & Unclear & Unclear & Yes \\
\hline
\end{tabular}




\section{REFERENCES}

Alavioon G, Hotzy H, Nakhro K, Rudolf S, Scofield DG, et al. 2017. Haploid selection within a single ejaculate increases offspring fitness. Proc. Natl. Acad. Sci. USA. 114:8053-58

Ardon F, Helms D, Sahin E, Bollwein H, Topfer-Petersen E, Waberski D. 2008. Chromatin-unstable boar spermatozoa have little chance of reaching oocytes in vivo. Reproduction. 135(4):461-70

Armbruster WS, Gobeille Rogers D. 2004. Does pollen competition reduce the cost of inbreeding? Am. J. Bot. 91(11):1939-43

Arnqvist G, Rowe L. 2005. Sexual Conflict. Princeton: University Press

Aronen T, Nikkanen T, Harju A, Tiimonen H, Häggman H. 2002. Pollen competition and seed-siring success in Picea abies. Theor. Appl. Genet. 104(4):638-42

Arunkumar R, Josephs EB, Williamson RJ, Wright SI. 2013. Pollen-specific, but not sperm-specific, genes show stronger purifying selection and higher rates of positive selection than sporophytic genes in Capsella grandiflora. Mol. Biol. Evol. 30:2475-86

Bachtrog D, Mank JE, Peichel CL, Otto SP, Kirkpatrick M, et al. 2014. Sex determination: Why so many ways of doing it? PLoS Biol. 12:001899

Ballard JWO, Whitlock MC. 2004. The incomplete natural history of mitochondria. Mol. Ecol. 13(4):729-44

Barr CM, Neiman M, Taylor DR. 2005. Inheritance and recombination of mitochondrial genomes in plants, fungi and animals: Research review. New Phytol. 168(1):39-50

Borg M, Brownfield L, Twell D. 2009. Male gametophyte development: a molecular perspective. J. Exp. Bot. 60(5):1465-78 
Borowsky R, Luk A, Kim RS. 2018. Unique sperm haplotypes are associated with phenotypically different sperm subpopulations in Astyanax fish. BMC Biol. 16:72

Brockdorff N, Turner BM. 2015. Dosage Compensation in Mammals. Cold Spring Harb. Perspect. Biol. 7(3):a019406

Caldwell KA, Handel MA. 1991. Protamine transcript sharing among postmeiotic spermatids. Proc. Natl. Acad. Sci. 88(6):2407-11

800 Carré D, Sardet C. 1984. Fertilization and early development in Beroe ovata. Dev. Biol. 105:188-95

Charlesworth B. 1978. Model for evolution of Y-chromosomes and dosage compensation. Proc. Natl. Acad. Sci. USA. 75:5618-5622

Charlesworth B. 1996. The evolution of chromosomal sex determination and dosage compensation. Curr. Biol. 6(2):149-62

Charlesworth B, Charlesworth D. 1987. Inbreeding depression and its evolutionary consequences. Annu. Rev. Ecol. Syst. 18(237-268):

Charlesworth D, Charlesworth B. 1992. The effects of selection in the gametophytic stage on mutational load. Evolution. 46:703-20

810 Chess A. 2012. Mechanisms and consequences of widespread random monoallelic expression. Nat. Rev. Genet. 13:421

Clarke HJ, Khan TN, Siddique KHM. 2004. Pollen selection for chilling tolerance at hybridisation leads to improved chickpea cultivars. Euphytica. 139:65-74

815 Cohen J. 1967. Correlation between sperm "redundancy" and chiasma frequency. Nature. 215:362-63 
Cohen J. 1973. Cross-overs, sperm redundancy and their close association. Heredity. 31:408-13

Crow JF, Kimura M. 1965. Evolution of sexual and asexual populations. Am. Nat. 99:439-50

Crow JF, Kimura M. 1970. An Introduction to Population Genetics Theory. New York, Evanston and London: Harper \& Row

Day T, Bonduriansky R. 2004. Intralocus sexual conflict can drive the evolution of genomic imprinting. Genetics. 167:1537-46

825 de Vries J, Archibald JM. 2018. Chapter One - Plastid Autonomy vs Nuclear Control Over Plastid Function. In Advances in Botanical Research, Vol. 85, eds. S-M Chaw, RK Jansen, pp. 1-28. Academic Press

Dean R, Zimmer F, Mank JE. 2014. The Potential Role of Sexual Conflict and Sexual Selection in Shaping the Genomic Distribution of Mito-nuclear Genes. Genome Biol. Evol. 6(5):1096-1104

Domínguez E, Cuartero J, Fernández-Muñoz R. 2005. Breeding tomato for pollen tolerance to low temperatures by gametophytic selection. Euphytica. 142(3):253-63

Dym M, Fawcett DW. 1971. Further Observations on the Numbers of Spermatogonia, Spermatocytes, and Spermatids Connected by Intercellular Bridges in the Mammalian Testis1. Biol. Reprod. 4(2):195215

Erickson RP. 1973. Haploid gene expression versus meiotic drive: the relevance of intercellular bridges during spermatogenesis. Nature. New Biol. 243:210-12

Erickson RP. 1990. Post-meiotic gene expression. Trends Genet. 6(8):264-68 
Ewing E. 1977. Selection at the haploid and diploid phases: cyclical variation. Genetics. 87:195-208

Ezawa K, Innan H. 2013. Competition Between the Sperm of a Single Male Can Increase the Evolutionary Rate of Haploid Expressed Genes. Genetics. 194(3):709-19

Fitzpatrick JL, Baer B. 2011. Polyandry reduces sperm length variation in social insects. Evolution. 65(10):3006-12

Good JM, Nachman MW. 2005. Rates of Protein Evolution Are Positively 850 Correlated with Developmental Timing of Expression During Mouse Spermatogenesis. Mol. Biol. Evol. 22(4):1044-52

Good JM, Vanderpool D, Smith KL, Nachman MW. 2011. Extraordinary Sequence Divergence at Tsga8, an X-linked Gene Involved in Mouse Spermiogenesis. Mol. Biol. Evol. 28(5):1675-86

855 Gordo I, Charlesworth B. 2000. The degeneration of asexual haploid populations and the speed of Muller's ratchet. Genetics. 154(3):137987

Gossmann TI, Schmid MW, Grossniklaus U, Schmid KJ. 2014. SelectionDriven Evolution of Sex-Biased Genes Is Consistent with Sexual Selection in Arabidopsis thaliana. Mol. Biol. Evol. 31(3):574-83

Gur Y, Breitbart H. 2006. Mammalian sperm translate nuclear-encoded proteins by mitochondrial-type ribosomes. Genes Dev. 20:411-16

Gur Y, Breitbart H. 2008. Protein synthesis in sperm: Dialog between mitochondria and cytoplasm. Mol Cell Endocrinol. 282(1-2):45-55

865 Haig D. 2000. The kinship theory of genomic imprinting. Annu. Rev. Ecol. Syst. 31:9-32 
Haldane JBS. 1932. The Causes of Evolution. New York: Harper

Hartl DL. 1976. Applications of meiotic drive in animal breeding and population control. In Proc. Intern. Congrecs Quant. Genetics, pp. 6388. Ames: lowa State Univeristy Press

Holt WV, Van Look KJW. 2004. Concepts in sperm heterogeneity, sperm selection and sperm competition as biological foundations for laboratory tests of semen quality. Reproduction. 127:527-35

Honys D, Twell D. 2004. Transcriptome analyses of haploid male gametophyte development in Arabidopsis. Genome Biol. 5:R85

Hough J, Immler S, Barrett SCH, Otto SP. 2013. Evolutionarily stable sex ratios and mutation load. Evolution. 67(7):1915-1925

Hourcade JD, Pérez-Crespo M, Fernández-González R, Pintado B, GutiérrezAdán A. 2010. Selection against spermatozoa with fragmented DNA after postovulatory mating depends on the type of damage. Reprod. Biol. Endocrinol. RBE. 8:9-9

Immler S, Arnqvist G, Otto SP. 2012. Ploidally antagonistic selection maintains stable genetic polymorphism. Evolution. 66(1):55-65

Immler S, Otto SP. 2014. Driven Apart: The Evolution of Ploidy Differences between the Sexes under Antagonistic Selection. Am. Nat. 183(1):96107

Kanippayoor RL, Alpern JHM, Moehring AJ. 2013. Protamines and spermatogenesis in Drosophila and Homo sapiens. Spermatogenesis. 3(2):e24376

890 Kirkpatrick M. 1994. The Evolution of Haploid-Diploid Life Cycles, Vol. 25. Providence, Rhode Island, USA: American Mathematical Society 
Kondrashov AS, Crow JF. 1991. Haploidy or diploidy: which is better? Nature. 351(6324):314-15

Lankinen A, Maad J, Armbruster WS. 2009. Pollen-tube growth rates in Collinsia heterophylla (Plantaginaceae): One-donor crosses reveal heritability but no effect on sporophytic-offspring fitness. Ann Bot Lond. 103(9):941-50

Lenormand T. 2003. The evolution of sex dimorphism in recombination. Genetics. 163:811-22

900 Lenormand T, Dutheil J. 2005. Recombination difference between sexes: A role for haploid selection. PLoS Biol. 3(3):e63

Lenz TL, Hafer N, Samonte IE, Yeates SE, Milinski M. 2018. Cryptic haplotype-specific gamete selection yields offspring with optimal $\mathrm{MHC}$ immune genes: sperm selection for optimal immune genes. Evolution. 72(11):2478-90

Lindsley DL, Grell EH. 1969. Spermiogenesis without chromosomes in Drosophila melanogaster. Genetics. 61:69-78

Losdat S, Chang S-M, Reid JM. 2014. Inbreeding depression in male gametic performance. J. Evol. Biol. 27(6):992-1011

910 Lu S, Zong C, Fan W, Yang M, Li J, et al. 2012. Probing Meiotic Recombination and Aneuploidy of Single Sperm Cells by WholeGenome Sequencing. Science. 338(6114):1627

Lyon MF, Glenister PH, Hawker SG. 1972. Do the H-2 and T-Loci of the Mouse have a Function in the Haploid Phase of Sperm? Nature. 240:152 
Mable BK, Otto SP. 1998. The evolution of life cycles with haploid and diploid phases. BioEssays. 20:453-62

MacLaughlin J, Terner C. 1973. Ribonucleic acid synthesis by spermatozoa from the rat and hamster. Biochem. J. 133(4):635-39

920 Manning JT, Chamberlain AT. 1994. Sib competition and sperm competitiveness: an answer to "Why so many sperm?" and the recombination/sperm number correlation. Proc. R. Soc. Lond. Ser. B. 256:177-82

Mascarenhas JP. 1990. Gene activity during pollen development. Annu. Rev. Plant Physiol. Plant Mol. Biol. 41:317-38

Miller D, Ostermeier GC. 2006. Towards a better understanding of RNA carriage by ejaculate spermatozoa. Hum. Reprod. Update. 12(6):75767

Mulcahy DL. 1975. Gamete Competition in Plants and Animals. Amsterdam: North Holland Publishing Company

Muller HJ, Settles F. 1927. The non-functioning of the genes in spermatozoa. Z Indukt Astamm Verebunsl. 43:285-312

Nadeau JH. 2017. Do gametes woo? Evidence for non-random unions at fertilization. Genetics. 207(2):369-87

935 Orr HA, Otto SP. 1994. Does diploidy increase the rate of adaptation? Genetics. 136:1475-80

Otto SP, Scott MF, Immler S. 2015. Evolution of haploid selection in predominantly diploid organisms. Proc. Natl. Acad. Sci. USA. $112: 15952-15957$ 
940 Park C, Carrel L, Makova KD. 2010. Strong Purifying Selection at Genes Escaping X Chromosome Inactivation. Mol. Biol. Evol. 27(11):2446-50 Parker GA. 1993. Sperm competition games: sperm size and sperm number under adult control. Proc. R. Soc. Lond. Ser. B. 253:245-54

Parker GA, Begon ME. 1993. Sperm competition games: sperm size and 945 number under gametic control. Proc. R. Soc. Lond. Ser. B. 253:255-62

Pitnick S, Dobler R, Hosken DJ. 2009. Sperm length is not influenced by haploid gene expression in the flies Drosophila melanogaster and Scathophaga stercoraria. Proc. R. Soc. B-Biol. Sci. 276:4029-34

Podlaha O, Webb DM, Tucker PK, Zhang J. 2005. Positive Selection for Indel 950 Substitutions in the Rodent Sperm Protein Catsper1. Mol. Biol. Evol. 22(9):1845-52

Podlaha O, Zhang J. 2003. Positive selection on protein-length in the evolution of a primate sperm ion channel. Proc. Natl. Acad. Sci. USA. 100(21):12241-46

955 Premkumar E, Bhargava PM. 1972. Transcription and Translation in Bovine Spermatozoa. Nature. New Biol. 240(100):139-43

Promerová M, Alavioon G, Tusso S, Burri R, Immler S. 2017. No evidence for MHC class II-based non-random mating at the gametic haplotype in Atlantic salmon. Heredity. 118:563-67

960 Radzvilavicius AL, Hadjivasiliou Z, Pomiankowski A, Lane N. 2016. Selection for Mitochondrial Quality Drives Evolution of the Germline. PLOS Biol. 14(12):e2000410

Ramm SA, Scharer L, Ehmcke J, Wistuba J. 2014. Sperm competition and the evolution of spermatogenesis. Mol. Hum. Reprod. 20(12):1169-79 
965 Reed F, Aquadro C. 2006. Mutation, selection and the future of human evolution. Trends Genet. 22(9):479-84

Reik W, Walter J. 2001. Genomic imprinting: parental influence on the genome. Nat. Rev. Genet. 2(1):21-32

Romiguier J, Lourenco J, Gayral P, Faivre N, Weinert LA, et al. 2014. Population genomics of eusocial insects: the costs of a vertebrate-like effective population size. J. Evol. Biol. 27(3):593-603

Rossignol R, Faustin B, Rocher C, Malgat M, Mazat J-P, Letellier T. 2003. Mitochondrial threshold effects. Biochem. J. 370(3):751-62

Sandler G, Beaudry FEG, Barrett SCH, Wright SI. 2018. The effects of haploid selection on $\mathrm{Y}$ chromosome evolution in two closely related dioecious plants. Evol. Lett. 2(4):368-77

Sari-Gorla M, Frova C. 1997. Pollen Tube Growth and Pollen Selection Improvement. New Yrok, USA: Cambridge University Press

Scott C, de Souza FF, Aristizabal VHV, Hethrington L, Krisp C, et al. 2018a. Proteomic profile of sex-sorted bull sperm evaluated by SWATH-MS analysis. Anim. Reprod. Sci. 198:121-28

Scott MF, Osmond MM, Otto SP. 2018b. Haploid selection, sex ratio bias, and transitions between sex-determining systems. PLOS Biol. 16(6):e2005609

985 Scott MF, Otto SP. 2017. Haploid Selection Favours Suppressed Recombination Between Sex Chromosomes Despite Causing Biased Sex Ratios. Genetics. 1631-49

Scudo FM. 1967. Selection on both haplo and diplophase. Genetics. 56:693704 
990 Sharp NP, Sandell L, James CG, Otto SP. 2018. The genome-wide rate and spectrum of spontaneous mutations differ between haploid and diploid yeast. Proc. Natl. Acad. Sci. 115(22):E5046-55

Shoubridge EA, Wai T. 2007. Mitochondrial DNA and the Mammalian Oocyte. In Current Topics in Developmental Biology, Vol. 77, pp. 87-111.

995 Elsevier

Singh ND, Koerich LB, Carvalho AB, Clark AG. 2014. Positive and Purifying Selection on the Drosophila Y Chromosome. Mol. Biol. Evol. $31(10): 2612-23$

Singh ND, Larracuente AM, Clark AG. 2008. Contrasting the Efficacy of 1000 Selection on the $\mathrm{X}$ and Autosomes in Drosophila. Mol. Biol. Evol. 25(2):454-67

Snook RR, Hosken DJ, Karr TL. 2011. The biology and evolution of polyspermy: insights from cellular and functional studies of sperm and centrosomal behavior in the fertilized egg. REPRODUCTION. 142(6):779-92

Snow AA, Spira TP. 1996. Pollen-tube competition and male fitness in Hibiscus moscheutos. Evolution. 50(5):1866-70

Spillane C, Schmid KJ, Laoueillé-Duprat S, Pien S, Escobar-Restrepo J-M, et al. 2007. Positive darwinian selection at the imprinted MEDEA locus in plants. Nature. $448: 349$

Strasburger E. 1894. The periodic reduction of the number of the chromosomes in the life-history of living organisms. Ann. Bot. 8:281316 
Strasburger E. 1905. Typische und Allotypische Kernteilung. Ergebnisse und Erörterungen. In Histologische Beiträge Zur Vererbungsfrage, Vol. 42, p. 62. Gebrüder Berntraeger

Swanson WJ, Vacquier VD. 2002. The rapid evolution of reproductive proteins. Nat. Rev. Genet. 3:137-44

Walbot V, Evans MM. 2003. Unique features of the plant life cycle and their consequences. Nat. Rev. Genet. 4(5):369

Wang J, Fan HC, Behr B, Quake SR. 2012. Genome-wide single cell analysis of recombination activity and de novo mutation rates in human sperm. Cell. 150(2):402-12

Wang S, Zhang L, Meyer E, Matz MV. 2009. Construction of a high-resolution genetic linkage map and comparative genome analysis for the reefbuilding coral Acropora millepora. Genome Biol. 10(11):R126

Ward WS. 2018. Organization of sperm DNA by the nuclear matrix. Am. J. Clin. Exp. Urol. 6(2):87-92

Wedekind C, Chapuisat M, Macas E, Rülicke T. 1996. Non-random fertilization in mice correlates with the $\mathrm{MHC}$ and something else. Heredity. 77:400-409

Wedekind C, Walker M, Portmann J, Cenni B, Muller R, Binz T. 2004. MHClinked susceptibility to a bacterial infection, but no MHC-linked cryptic female choice in whitefish. J. Evol. Biol. 17:11-18

1035 Xu S, Ackerman MS, Long H, Bright L, Spitze K, et al. 2015. A Male-Specific Genetic Map of the Microcrustacean Daphnia pulex Based on SingleSperm Whole-Genome Sequencing. Genetics. 201(1):31-38 
Zamir D, Vallejos EC. 1983. Temperature effects on haploid selection of tomato microscopres and pollen grains. In Pollen: Biology and

1040 Implications for Plant Breeding, eds. DL Mulcahy, E Ottaviano, pp. 335-42. New York: Elsevier

Zheng Y, Deng X, Martin-DeLeon PA. 2001. Lack of sharing of Spam1 (Ph20) among mouse spermatids and transmission ratio distortion. Biol. Reprod. 64(6):1730-38

1045 